\title{
ON EXISTENCE AND A DOMINATED CONVERGENCE THEOREM FOR WEIGHTED $g$-SUMMABILITY
}

\author{
FRED M. WRIGHT AND MELVIN L. KLASI
}

\begin{abstract}
Let $\left(w_{1}, w_{2}, w_{3}\right)$ be an ordered triple of real numbers such that $w_{1}+w_{2}+w_{3}=1$. Let $g$ be a real-valued function on the entire real axis which is of bounded variation on every closed interval. For $f$ a real-valued function on the entire real axis which is bounded on a closed interval $[a, b]$, we use the F. Riesz step function approach to define the concept of $f$ being $\left(w_{1}, w_{2}, w_{3}\right)$ $g$-summable over $[a, b]$, and we define the integral

$$
\left[F,\left(w_{1}, w_{2}, w_{3}\right)\right] s \int_{a}^{b} f(x) d g(x)
$$

when $f$ has this property. We show that this integral extends the weighted refinement integral $\left[F,\left(w_{1}, w_{2}, u_{3}\right)\right] \int_{a}^{b} f(x) d g(x)$ for $f$ 's as above. This paper generalizes the method of Pasquale Porcelli for the Stieltjes mean sigma integral. We present an existence theorem for the integral defined here involving saltus and continuous parts of $g$. We establish a convergence theorem for this integral which is analogous to the Lebesgue Dominated Convergence Theorem for the Lebesgue-Stieltjes integral.
\end{abstract}

1. Introduction. Let $\left(w_{1}, w_{2}, w_{3}\right)$ be an ordered triple of real numbers such that $w_{1}+w_{2}+w_{3}=1$. Let $g$ be a real-valued function on the entire real axis which is of bounded variation on every closed interval. We let $g_{s}$ be a saltus function and $g_{c}$ be a continuous function on the entire real axis such that $g=g_{s}+g_{c}$. For $f$ a real-valued function on the entire real axis which is bounded on the closed interval $[a, b]$, we use the F. Riesz step function approach to define the concept of $f$ being $\left(w_{1}, w_{2}, w_{3}\right) g$-summable over $[a, b]$, and we define the integral

$$
\left[F,\left(w_{1}, w_{2}, w_{3}\right)\right] s \int_{a}^{b} f(x) d g(x)
$$

Received by the editors October 6, 1971.

AMS 1970 subject classifications. Primary 26A42, 28A25; Secondary 26A15, 26A45, 28A10, 28A20.

Key words and phrases. Step function, bounded variation, Stieltjes mean sigma integral, F. Riesz step function approach, saltus function, continuous function, weighted refinement integral, Lebesgue-Stieltjes integral, $\left(w_{1}^{\prime}, w_{2}, w_{3}\right) g$-summable, Lebesgue Dominated Convergence Theorem, iterated limits, Banach space. 
when $f$ has this property. This integral extends the weighted refinement integral

$$
\left[F,\left(w_{1}, w_{2}, w_{3}\right)\right] \int_{a}^{b} f(x) d g(x)
$$

of [1] for $f$ 's as here. This paper generalizes the method of Porcelli [2] for the Stieltjes mean sigma integral.

We show that a function $f$ as above is $\left(w_{1}, w_{2}, w_{3}\right) g$-summable over $[a, b]$ iff the weighted refinement integral

$$
\left[F,\left(w_{1}, w_{2}, w_{3}\right)\right] \int_{a}^{b} f(x) d g_{s}(x)
$$

and the Lebesgue-Stieltjes integral

$$
\text { (LS) } \int_{[a, b]} f(x) d g_{c}(x)
$$

exist. Furthermore, we show that when (1.1) exists it is the sum of (1.3) and (1.4). We then verify that if the weighted refinement integral (1.2) exists, then $f$ is $\left(w_{1}, w_{2}, w_{3}\right) g$-summable over $[a, b]$, and (1.2) equals (1.1). We then point out that for certain $g$ 's the function $f$ may be $\left(w_{1}, w_{2}, w_{3}\right)$ $g$-summable over $[a, b]$ when (1.2) does not exist.

We establish a result for (1.1) which is analogous to the Lebesgue Dominated Convergence Theorem for the Lebesgue-Stieltjes integral.

2. Definitions. In the rest of this paper, all functions are real-valued functions on the entire real axis. Also, $\left(w_{1}, w_{2}, w_{3}\right)$ is an ordered triple of real numbers such that $w_{1}+w_{2}+w_{3}=1$, and $g$ is a real-valued function on the entire real axis of bounded variation on every closed interval. We let $g_{s}$ be a saltus function and $g_{c}$ be a continuous function on the entire real axis such that $g=g_{s}+g_{c}$.

Definition 2.1. Let $g^{*}$ be the nondecreasing function such that $g^{*}(x)=$ $V(g,[x, 0])$ for $x<0, g^{*}(0)=0$, and $g^{*}(x)=V(g,[0, x])$ for $x>0$. Let $f$ be a function bounded on the closed interval $[a, b]$. If either $w_{2}$ or $w_{3}$ is different from 0 , suppose $f\left(x^{+}\right)$exists for all $x$ in $[a, b)$ such that $g\left(x^{+}\right) \neq$ $g(x)$; if either $w_{1}$ or $w_{2}$ is different from 0 , suppose $f\left(x^{-}\right)$exists for all $x$ in $(a, b]$ such that $g\left(x^{-}\right) \neq g(x)$. Suppose there is a sequence $\left\{f_{n}\right\}_{n=1}^{\infty}$ of step functions uniformly bounded on $[a, b]$ such that:

(i) $\lim _{n \rightarrow \infty} f_{n}(x)=f(x)$ for all $x$ in $[a, b]$ except for a subset of $[a, b]$ of outer $g^{*}$-measure 0 ;

(ii) if either $w_{2}$ or $w_{3}$ is different from $0, \lim _{n \rightarrow \infty} f_{n}\left(x^{+}\right)=f\left(x^{+}\right)$for all $x$ in $[a, b)$ such that $g\left(x^{+}\right) \neq g(x)$; 
(iii) if either $w_{1}$ or $w_{2}$ is different from $0, \lim _{n \rightarrow \infty} f_{n}\left(x^{-}\right)=f\left(x^{-}\right)$for all $x$ in $(a, b]$ such that $g\left(x^{-}\right) \neq g(x)$.

Then, we say that $f$ is $\left(w_{1}, w_{2}, w_{3}\right) g$-summable over $[a, b]$.

THEOREM 2.1. Let $f$ be a function that is $\left(w_{1}, w_{2}, w_{3}\right)$ g-summable over $[a, b]$. Let $\left\{f_{n}\right\}_{n=1}^{\infty}$ be a sequence of step functions as in Definition 2.1. Then, the Lebesgue-Stieltjes integral

$$
(\mathrm{LS}) \int_{[a, b]} f(x) d g(x)
$$

exists. Moreover,

exists and equals

$$
\lim _{n \rightarrow \infty}\left[F,\left(w_{1}, w_{2}, w_{3}\right)\right] \int_{a}^{b} f_{n}(x) d g(x)
$$

$$
\begin{aligned}
(\mathrm{LS}) \int_{[a, b]} f(x) d g(x)+ & \left\{\left(1-w_{1}\right) \sum_{x \in[a, b)}\left[f\left(x^{+}\right)-f(x)\right]\left[g\left(x^{+}\right)-g(x)\right]\right. \\
& -\left(1-w_{3}\right) \sum_{x \in(a, b]}\left[f(x)-f\left(x^{-}\right)\right]\left[g(x)-g\left(x^{-}\right)\right] \\
& \left.-f(b)\left[g\left(b^{+}\right)-g(b)\right]-f(a)\left[g(a)-g\left(a^{-}\right)\right]\right\} .
\end{aligned}
$$

Proof. By the Lebesgue Dominated Convergence Theorem, the Lebesgue-Stieltjes integral (2.1) exists, and

$$
\lim _{n \rightarrow \infty}(\mathrm{LS}) \int_{[a, b]} f_{n}(x) d g(x)=(\mathrm{LS}) \int_{[a, b]} f(x) d g(x) .
$$

From Theorem 2.5 in [1], we see that, for each positive integer $n$,

$$
\begin{aligned}
{\left[F,\left(w_{1}, w_{2}, w_{3}\right)\right] \int_{a}^{b} f_{n}(x) d g(x) } & \\
=(\mathrm{LS}) \int_{[a, b]} f_{n}(x) d g(x)+ & \left\{\left(1-w_{1}\right) \sum_{x \in[a, b)}\left[f_{n}\left(x^{+}\right)-f_{n}(x)\right]\left[g\left(x^{+}\right)-g(x)\right]\right. \\
& -\left(1-w_{3}\right) \sum_{x \in(a, b]}\left[f_{n}(x)-f_{n}\left(x^{-}\right)\right]\left[g(x)-g\left(x^{-}\right)\right] \\
& \left.-f_{n}(b)\left[g\left(b^{+}\right)-g(b)\right]-f_{n}(a)\left[g(a)-g\left(a^{-}\right)\right]\right\} .
\end{aligned}
$$

Taking the limit of the preceding expression as $n \rightarrow \infty$, we have that the limit (2.2) exists and equals (2.3).

This completes the proof of Theorem 2.1.

Now we can define the extended integral (1.1). 
Definition 2.2. Let $f$ be a function which is $\left(w_{1}, w_{2}, w_{3}\right) g$-summable over $[a, b]$. Let $\left\{f_{n}\right\}_{n=1}^{\infty}$ be a sequence of step functions as in Definition 2.1. Then, we let

$$
\left[F,\left(w_{1}, w_{2}, w_{3}\right)\right] s \int_{a}^{b} f(x) d g(x)
$$

denote the limit

$$
\lim _{n \rightarrow \infty}\left[F,\left(w_{1}, w_{2}, w_{3}\right)\right] \int_{a}^{b} f_{n}(x) d g(x) .
$$

It follows from Theorem 2.1 that the extended integral (1.1) is independent of the particular sequence of step functions used in Definition 2.2 having the properties there. Moreover, this integral is equal to (2.3).

3. Existence. The first theorem of this section provides necessary conditions for a function $f$ to be $\left(w_{1}, w_{2}, w_{3}\right) g$-summable over a closed interval $[a, b]$ of the real axis. This theorem also gives the extended integral (1.1) as the sum of the weighted refinement integral (1.3) and the Lebesgue-Stieltjes integral (1.4).

THEOREM 3.1. Let $f$ be a function which is $\left(w_{1}, w_{2}, w_{3}\right)$ g-summable over $[a, b]$. Then, the weighted refinement integral

$$
\left[F,\left(w_{1}, w_{2}, w_{3}\right)\right] \int_{a}^{b} f(x) d g_{s}(x)
$$

and the Lebesgue-Stieltjes integral

$$
(\mathrm{LS}) \int_{[a, b]} f(x) d g_{c}(x)
$$

exist. Moreover, the extended integral (1.1) is equal to the sum of (1.3) and (1.4).

Proof. The existence of the weighted refinement integral (1.3) follows from Theorem 2.3 in [1]. The existence of the Lebesgue-Stieltjes integral (1.4) follows from the Lebesgue Dominated Convergence Theorem. From Theorem 2.5 in [1] we have that (1.3) equals

$$
\begin{aligned}
(\mathrm{LS}) \int_{[a, b]} f(x) d g_{s}(x)+ & \left\{\left(1-w_{1}\right) \sum_{x \in[a, b)}\left[f\left(x^{+}\right)-f(x)\right]\left[g\left(x^{+}\right)-g(x)\right]\right. \\
& -\left(1-w_{3}\right) \sum_{x \in(a, b]}\left[f(x)-f\left(x^{-}\right)\right]\left[g(x)-g\left(x^{-}\right)\right] \\
& \left.-f(b)\left[g\left(b^{+}\right)-g(b)\right]-f(a)\left[g(a)-g\left(a^{-}\right)\right]\right\} .
\end{aligned}
$$


Adding (1.4) to the preceding, we obtain the expression (2.3) which equals (1.1).

This completes the proof of Theorem 3.1.

The next theorem is the converse of Theorem 3.1 and is our main result here.

THEOREM 3.2. Let $f$ be a function bounded on the closed interval $[a, b]$. Suppose the weighted refinement integral (1.3) and the Lebesgue-Stieltjes integral (1.4) exist. Then, $f$ is $\left(w_{1}, w_{2}, w_{3}\right)$ g-summable over $[a, b]$.

Proof. In view of Theorems 2.1 and 2.2 in [1] and their analogues for left-hand limits, the existence of the weighted refinement integral (1.3) implies that :

(a) if either $w_{2}$ or $w_{3}$ is different from $0, f\left(x^{+}\right)$exists for all $x$ in $[a, b)$ such that $g\left(x^{+}\right) \neq g(x)$;

(b) if either $w_{1}$ or $w_{2}$ is different from $0, f\left(x^{-}\right)$exists for all $x$ in $(a, b]$ such that $g\left(x^{-}\right) \neq g(x)$.

The existence of the Lebesgue-Stieltjes integral (1.4) implies the existence of the Lebesgue-Stieltjes integral

$$
\text { (LS) } \int_{[a, b]} f(x) d g(x) \text {. }
$$

Therefore, there is a sequence $\left\{f_{n}\right\}_{n=1}^{\infty}$ of step functions uniformly bounded on $[a, b]$ such that $\lim _{n \rightarrow \infty} f_{n}(x)=f(x)$ for all $x$ in $[a, b]$ except for a subset of $[a, b]$ of outer $g^{*}$-measure 0 .

Suppose now that either $w_{2}$ or $w_{3}$ is different from 0 . Consider first the case where there is a one-to-one sequence $\left\{x_{j, 1}\right\}_{j=1}^{\infty}$ whose range consists of all points $x$ of $[a, b)$ such that $g\left(x^{+}\right) \neq g(x)$. For each positive integer $n$, let the points $x_{1,1}, x_{2,1}, \cdots, x_{n, 1}$ be ordered as $\zeta_{1, n, 1}<\zeta_{2, n, 1}<\cdots<\zeta_{n, n, 1}$, and let $\delta_{n, 1}$ be a positive real number less than

$$
\min \left\{\zeta_{2, n, 1}-\zeta_{1, n, 1}, \zeta_{3, n, 1}-\zeta_{2, n, 1}, \cdots, \zeta_{n, n, 1}-\zeta_{n-1, n, 1}, b-\zeta_{n, n, 1}\right\}
$$

such that, for each positive integer $j$ not exceeding $n,\left|f(x)-f\left(\zeta_{j, n, 1}^{+}\right)\right|<$ $1 / n$ for all $x$ in $\left(\zeta_{j, n, 1}, \zeta_{j, n, 1}+\delta_{n, 1}\right]$. For each positive integer $n$, let $f_{n, 1}$ be the step function such that

$$
\begin{array}{ll}
f_{n, 1}(x)=f\left(\zeta_{j, n, 1}^{+}\right) & \text {for } j \text { a positive integer not exceeding } n \\
f_{n, 1}(x)=f_{n}(x) & \text { for all other } x .
\end{array}
$$

For each positive integer $j$, we have that $f_{n, 1}\left(x_{j, 1}^{+}\right)=f\left(x_{j, 1}^{+}\right)$for all integers $n \geqq j$, and thus it follows that $\lim _{n \rightarrow \infty} f_{n, 1}\left(x_{j, 1}^{+}\right)=f\left(x_{j, 1}^{+}\right)$. Next we show that 
if $x^{*}$ is a point of $[a, b]$ such that $\lim _{n \rightarrow \infty} f_{n}\left(x^{*}\right)=f\left(x^{*}\right)$, then

$$
\lim _{n \rightarrow \infty} f_{n, 1}\left(x^{*}\right)=f\left(x^{*}\right) .
$$

Let $\varepsilon$ be any given positive real number. Let $n^{\prime}$ be a positive integer such that $1 / n^{\prime}<\varepsilon$ and such that $\left|f_{n}\left(x^{*}\right)-f\left(x^{*}\right)\right|<\varepsilon$ for all integers $n>n^{\prime}$. Let $n$ be any particular integer such that $n>n^{\prime}$. If there is a positive integer $j$ not exceeding $n$ such that $x^{*}$ is in $\left(\zeta_{j, n, 1}, \zeta_{j, n, 1}+\delta_{n, 1}\right]$, then

$$
\left|f_{n, 1}\left(x^{*}\right)-f\left(x^{*}\right)\right|=\left|f\left(\zeta_{j, n, 1}^{+}\right)-f\left(x^{*}\right)\right|<1 / n<\varepsilon .
$$

If there is no such $j$, then

$$
\left|f_{n, 1}\left(x^{*}\right)-f\left(x^{*}\right)\right|=\left|f_{n}\left(x^{*}\right)-f\left(x^{*}\right)\right|<\varepsilon .
$$

Thus, $\lim _{n \rightarrow \infty} f_{n, 1}\left(x^{*}\right)=f\left(x^{*}\right)$. For the case where there is a one-to-one finite sequence $\left\{x_{j, 1}\right\}_{j=1}^{J}$ whose range consists of all points $x$ of $[a, b)$ such that $g\left(x^{+}\right) \neq g(x)$, proceed in a manner similar to the above to determine a sequence $\left\{f_{n, 1}\right\}_{n=1}^{\infty}$ of step functions. If $g\left(x^{+}\right)=g(x)$ for all $x$ in $[a, b)$, let $f_{n, 1}=f_{n}$ for every positive integer $n$.

If $w_{2}=0=w_{3}$, let $f_{n, 1}=f_{n}$ for every positive integer $n$.

Suppose next that at least one of $w_{1}, w_{2}$ is different from 0 and that there is at least one $x$ in $(a, b]$ such that $g\left(x^{-}\right) \neq g(x)$. We use the sequence $\left\{f_{n, 1}\right\}_{n=1}^{\infty}$ to determine a sequence $\left\{f_{n, 2}\right\}_{n=1}^{\infty}$ of step functions uniformly bounded on $[a, b]$ and having the following two properties:

(1) $\lim _{n \rightarrow \infty} f_{n, 2}(x)=f(x)$ for all $x$ in $[a, b]$ except for a subset of $[a, b]$ of outer $g^{*}$-measure 0 ;

(2) $\lim _{n \rightarrow \infty} f_{n, 2}\left(x^{-}\right)=f\left(x^{-}\right)$for all $x$ in $(a, b]$ such that $g\left(x^{-}\right) \neq g(x)$.

Our method is similar to the one employed above in using the sequence $\left\{f_{n}\right\}_{n=1}^{\infty}$ to determine the sequence $\left\{f_{n, 1}\right\}_{n=1}^{\infty}$ for the case where at least one of $w_{2}, w_{3}$ is different from 0 and where there is at least one $x$ in $[a, b)$ such that $g\left(x^{+}\right) \neq g(x)$.

Suppose then either that $w_{1}=0=w_{2}$ or that $g\left(x^{-}\right)=g(x)$ for all $x$ in $(a, b]$. We let $f_{n, 2}=f_{n, 1}$ for every positive integer $n$. We note that $\left\{f_{n, 2}\right\}_{n=1}^{\infty}$ is a sequence of step functions uniformly bounded on $[a, b]$ and having the property that $\lim _{n \rightarrow \infty} f_{n, 2}(x)=f(x)$ for all $x$ in $[a, b]$ except for a subset of $[a, b]$ of outer $g^{*}$-measure 0 .

It now remains to be shown that if either $w_{2}$ or $w_{3}$ is different from 0 , then $\lim _{n \rightarrow \infty} f_{n, 2}\left(x^{+}\right)=f\left(x^{+}\right)$for all $x$ in $[a, b)$ such that $g\left(x^{+}\right) \neq g(x)$. So, suppose that either $w_{2}$ or $w_{3}$ is different from 0 . We treat the case where at least one of $w_{1}, w_{2}$ is different from 0 and where there is a one-to-one sequence $\left\{x_{j, 2}\right\}_{j=1}^{\infty}$ whose range consists of all points $x$ of $(a, b]$ such that $g\left(x^{-}\right) \neq g(x)$. For each positive integer $n$, let the points $x_{1,2}, x_{2,2}, \cdots, x_{n, 2}$ be ordered as $\zeta_{1, n, 2}<\zeta_{2, n, 2}<\cdots<\zeta_{n, n, 2}$, and let $\delta_{n, 2}$ be a positive real 
number less than

$$
\min \left\{\zeta_{1, n, 2}-a, \zeta_{2, n, 2}-\zeta_{1, n, 2}, \zeta_{3, n, 2}-\zeta_{2, n, 2}, \cdots, \zeta_{n, n, 2}-\zeta_{n-1, n, 2}\right\}
$$

such that, for each positive integer $j$ not exceeding $n,\left|f(x)-f\left(\zeta_{\bar{j}, n, 2}\right)\right|<1 / n$ for all $x$ in $\left[\zeta_{j, n, 2}-\delta_{n, 2}, \zeta_{j, n, 2}\right)$. For each positive integer $n$, let $f_{n, 2}$ be the step function such that

$$
\begin{array}{ll}
f_{n, 2}(x)=f\left(\zeta_{j, n, 2}\right) & \text { for } j \text { a positive integer not exceeding } n \\
& \text { and } x \in\left[\zeta_{j, n, 2}-\delta_{n, 2}, \zeta_{j, n, 2}\right),
\end{array}
$$

Let $\tilde{x}$ be a point of $[a, b)$ such that $g\left(\tilde{x}^{+}\right) \neq g(\tilde{x})$. Let $\varepsilon$ be any given positive real number. Let $n^{\prime \prime}$ be a positive integer such that $2 / n^{\prime \prime}<\varepsilon$ and such that $\left|f_{n, 1}\left(\tilde{x}^{+}\right)-f\left(\tilde{x}^{+}\right)\right|<\varepsilon$ for all integers $n>n^{\prime \prime}$. Let $n$ be any particular integer such that $n>n^{\prime \prime}$. If there is a positive integer $j$ not exceeding $n$ such that $\tilde{x}$ is in $\left[\zeta_{j, n, 2}-\delta_{n, 2}, \zeta_{j, n, 2}\right)$, there is a real number $\bar{x}$ satisfying $\tilde{x}<\bar{x}<\zeta_{j, n, 2}$ such that $\left|f(\bar{x})-f\left(\tilde{x}^{+}\right)\right|<1 / n$, and thus

$$
\begin{aligned}
\left|f_{n, 2}\left(\tilde{x}^{+}\right)-f\left(\tilde{x}^{+}\right)\right| & =\left|f\left(\zeta_{\overline{j, n, 2}}\right)-f\left(\tilde{x}^{+}\right)\right| \\
& \leqq\left|f\left(\zeta_{\overline{j, n, 2}}\right)-f(\bar{x})\right|+\left|f(\bar{x})-f\left(\tilde{x}^{+}\right)\right|<2 / n<\varepsilon .
\end{aligned}
$$

If there is no such $j$, then

$$
\left|f_{n, 2}\left(\tilde{x}^{+}\right)-f\left(\tilde{x}^{+}\right)\right|=\left|f_{n, 1}\left(\tilde{x}^{+}\right)-f\left(\tilde{x}^{+}\right)\right|<\varepsilon .
$$

Thus, $\lim _{n \rightarrow \infty} f_{n, 2}\left(\tilde{x}^{+}\right)=f\left(\tilde{x}^{+}\right)$.

This completes the proof of Theorem 3.2.

We conclude this section by showing that, for the given ordered triple $\left(w_{1}, w_{2}, w_{3}\right)$ of weights and the given integrator function $g$, the integral (1.1) extends the weighted refinement integral (1.2) for the class of integrand functions $f$ considered in Definition 2.1.

THEOREM 3.3. Let $f$ be a function bounded on the closed interval $[a, b]$ such that the weighted refinement integral (1.2) exists. Then, $f$ is $\left(w_{1}, w_{2}, w_{3}\right)$ g-summable over $[a, b]$, and (1.2) equals (1.1).

Proof. From Theorems 2.1 and 2.2 in [1], the analogues of these results for left-hand limits, and Theorem 2.3 in [1], we have that the weighted refinement integral (1.3) exists. We have from Theorem 2.5 of [1] that the Lebesgue-Stieltjes integral (1.4) exists. It then follows from Theorem 3.2 that $f$ is $\left(w_{1}, w_{2}, w_{3}\right) g$-summable over $[a, b]$. The fact that (1.2) equals (1.1) follows from Theorem 2.1 of this paper and Theorem 2.5 of $[1]$.

This completes the proof of Theorem 3.3. 
We present an example of a $g$ such that there exist $f$ 's which are $\left(w_{1}, w_{2}, w_{3}\right) g$-summable over $[a, b]$ but for which the weighted refinement integral (1.2) does not exist. It is easy to determine other such $g$ 's. Suppose that $g(x)=x$ for all real numbers $x$. Let $f$ be any function bounded and Lebesgue integrable on the closed interval $[a, b]$ of the real axis such that the Lebesgue measure of the set of discontinuities of $f$ on $[a, b]$ is greater than 0 . Then, $f$ is $\left(w_{1}, w_{2}, w_{3}\right) g$-summable over $[a, b]$, but the weighted refinement integral (1.2) does not exist.

4. Sequences of summable functions. The following theorem concerns the structure of the space of functions $f$ which are $\left(w_{1}, w_{2}, w_{3}\right) g$-summable over a given closed interval $[a, b]$ of the real axis. This theorem is a consequence of Theorems 3.1 and 3.2 of this paper, Theorem 2.3 of [1], the Lebesgue Dominated Convergence Theorem, and a classic iterated limits theorem.

THEOREM 4.1. Let $[a, b]$ be a given closed interval of the real axis. For each function $f$ which is $\left(w_{1}, w_{2}, w_{3}\right)$ g-summable over $[a, b]$, let the norm of $f$ be l.u.b. $\{|f(x)|: x \in[a, b]\}$. With this norm, the space of $\left(w_{1}, w_{2}\right.$, $w_{3}$ ) g-summable functions $f$ over $[a, b]$ is a Banach space.

We now use Theorem 3.2 to prove the following result for the integral (1.1) which is analogous to the Lebesgue Dominated Convergence Theorem for the Lebesgue-Stieltjes integral.

THEOREM 4.2. Let $f$ be a function bounded on the closed interval $[a, b]$. If $w_{2}$ or $w_{3}$ is different from 0 , suppose $f\left(x^{+}\right)$exists for all $x$ in $[a, b)$ such that $g\left(x^{+}\right) \neq g(x)$; if either $w_{1}$ or $w_{2}$ is different from 0 , suppose $f\left(x^{-}\right)$exists for all $x$ in $(a, b]$ such that $g\left(x^{-}\right) \neq g(x)$. Suppose there is a sequence $\left\{f_{n}\right\}_{n=1}^{\infty}$ of functions $\left(w_{1}, w_{2}, w_{3}\right)$ g-summable over $[a, b]$ and uniformly bounded on $[a, b]$ such that:

(i) $\lim _{n \rightarrow \infty} f_{n}(x)=f(x)$ for all $x$ in $[a, b]$ except for a subset of $[a, b]$ of outer $g^{*}$-measure 0 ;

(ii) if either $w_{2}$ or $w_{3}$ is different from $0, \lim _{n \rightarrow \infty} f_{n}\left(x^{+}\right)=f\left(x^{+}\right)$for all $x$ in $[a, b)$ such that $g\left(x^{+}\right) \neq g(x)$;

(iii) if either $w_{1}$ or $w_{2}$ is different from $0, \lim _{n \rightarrow \infty} f_{n}\left(x^{-}\right)=f\left(x^{-}\right)$for all $x$ in $(a, b]$ such that $g\left(x^{-}\right) \neq g(x)$.

Then, $f$ is $\left(w_{1}, w_{2}, w_{3}\right) g$-summable over $[a, b]$, and

$$
\lim _{n \rightarrow \infty}\left[F,\left(w_{1}, w_{2}, w_{3}\right)\right] s \int_{a}^{b} f_{n}(x) d g(x)=\left[F,\left(w_{1}, w_{2}, w_{3}\right)\right] s \int_{a}^{b} f(x) d g(x) .
$$

Proof. The existence of the weighted refinement integral (1.3) follows from Theorem 2.3 in [1]. The existence of the Lebesgue-Stieltjes integral 
(1.4) follows from Theorem 3.1 and the Lebesgue Dominated Convergence Theorem. We then have from Theorem 3.2 that the function $f$ is $\left(w_{1}, w_{2}, w_{3}\right)$ $g$-summable over $[a, b]$.

For each positive integer $n$,

$$
\begin{aligned}
{\left[F,\left(w_{1}, w_{2}, w_{3}\right)\right] s \int_{a}^{b} f_{n}(x) d g(x) } & \\
=(\mathrm{LS}) \int_{[a, b]} f_{n}(x) d g(x)+ & \left\{\left(1-w_{1}\right) \sum_{x \in[a, b)}\left[f_{n}\left(x^{+}\right)-f_{n}(x)\right]\left[g\left(x^{+}\right)-g(x)\right]\right. \\
- & \left(1-w_{3}\right) \sum_{x \in(a, b]}\left[f_{n}(x)-f_{n}\left(x^{-}\right)\right]\left[g(x)-g\left(x^{-}\right)\right] \\
& \left.-f_{n}(b)\left[g\left(b^{+}\right)-g(b)\right]-f_{n}(a)\left[g(a)-g\left(a^{-}\right)\right]\right\} .
\end{aligned}
$$

By the Lebesgue Dominated Convergence Theorem,

$$
\lim _{n \rightarrow \infty}(\mathrm{LS}) \int_{[a, b]} f_{n}(x) d g(x)=(\mathrm{LS}) \int_{[a, b]} f(x) d g(x) .
$$

If either $w_{2}$ or $w_{3}$ is different from 0 , we have as in the proof of Theorem 2.1 that

$$
\begin{aligned}
\lim _{n \rightarrow \infty} \sum_{x \in[a, b)}\left[f_{n}\left(x^{+}\right)-\right. & \left.f_{n}(x)\right]\left[g\left(x^{+}\right)-g(x)\right] \\
& =\sum_{x \in[a, b)}\left[f\left(x^{+}\right)-f(x)\right]\left[g\left(x^{+}\right)-g(x)\right] .
\end{aligned}
$$

If either $w_{1}$ or $w_{2}$ is different from 0 , we have similarly that

$$
\begin{aligned}
\lim _{n \rightarrow \infty} \sum_{x \in(a, b]}\left[f_{n}(x)-\right. & \left.f_{n}\left(x^{-}\right)\right]\left[g(x)-g\left(x^{-}\right)\right] \\
& =\sum_{x \in(a, b]}\left[f(x)-f\left(x^{-}\right)\right]\left[g(x)-g\left(x^{-}\right)\right] .
\end{aligned}
$$

Thus,

$$
\begin{aligned}
\lim _{n \rightarrow \infty}\left[F,\left(w_{1}, w_{2}, w_{3}\right)\right] s \int_{a}^{b} f_{n}(x) d g(x) & \\
& =\left[F,\left(w_{1}, w_{2}, w_{3}\right)\right] s \int_{a}^{b} f(x) d g(x) .
\end{aligned}
$$

This completes the proof of Theorem 4.2.

We close with the observation that there is a Gronwall inequality for the extended integral (1.1) like the Gronwall inequality for the weighted refinement integral established in [3]. 


\section{REFERENCES}

1. F. M. Wright and J. D. Baker, On integration-by-parts for weighted integrals, Proc. Amer. Math. Soc. 22(1969), 42-52. MR 39 \#7056.

2. P. Porcelli, Concerning integrals, Proc. Amer. Math. Soc. 5 (1954), 395-400. MR 15, 944; 1140.

3. F. M. Wright, M. L. Klasi and D. R. Kennebeck, The Gronwall inequality for w'eighted integrals, Proc. Amer. Math. Soc. 30 (1971), 504-510.

Department of Mathematics, Iowa State University, Ames, Iowa 50010

Department of Mathematics, South Dakota School of Mines and Technology, RAPID CITY, SOUTH DaKota 57701 